\title{
Variations of Ap stars in the Geneva system ${ }^{\star, \star \star}$
}

\author{
J. Manfroid ${ }^{1, \star \star \star}$, M. Burnet ${ }^{2}$, and P. Renson ${ }^{1}$ \\ 1 Institut d'Astrophysique, Université de Liège, avenue de Cointe 5, B-4000 Liège, Belgium \\ 2 Observatoire de Genève, CH-1290 Sauverny, Switzerland
}

Received March 4; accepted May 12, 1997

\begin{abstract}
The photometric behaviour of 21 Ap stars has been analyzed from observations obtained in the Geneva system. Variability has been detected for 9 Ap stars, as well as for a star which initially was a comparison star. Preliminary values for the periods are presented as well as an analytical description of the light curves.
\end{abstract}

Key words: stars: chemically peculiar; variable

\section{Introduction}

Short of spectrophotometric studies - which require extensive efforts and are currently unpractical and unthinkable at major observatories - intermediate-band photometry offers the best possibilities to characterize the light variations of Ap stars. Dedicated systems such as Maitzen's $\Delta a$ (Maitzen 1976) are certainly ideal since they offer direct measurement of physical parameters, but the Strömgren system has proven to be quite valuable in many studies (see, e.g., Vogt \& Faundez 1979; Mathys \& Manfroid 1985). This is also the case of the Geneva system which combines wide and intermediate bands (see, e.g., North 1984; North \& Adelman 1995).

In this paper we present observations of 21 Ap stars, most of which were never studied for variations. About half of them show clear optical variations. We derived the periods - not always unambiguously — and determined analytical model of the light curves in 17 colours and indices.

\footnotetext{
^ based on observations made at the Swiss telescope of the European Southern Observatory, La Silla, Chile.

** Complete Table 4 is available in electronic form at the CDS via anonymous ftp 130.79.128.5 or http://cdsweb.ustrasbg.fr/Abstract.html and full figures are accessible on the on-line version of the paper.

${ }^{\star \star \star}$ Research Director NFSR.
}

\section{The observational material}

The material has been collected during a 23-night observing run at La Silla with the $70 \mathrm{~cm}$ telescope of the Geneva Observatory equipped with the standard Geneva photometer (Burnet \& Rufener 1979). Each star was observed about once per night in the seven-colour Geneva photometric system $(U, B, V, B 1, B 2, V 1, G)$. Although the absolute photometry is of high quality, we observed Geneva standards as comparison or check stars in the vicinity of each programme stars.

Aside from the seven magnitudes we considered the usual color indices of the Geneva system. Moreover, we added the intermediate-band index $B 1-V 1$. Never used in Geneva photometry, this index is the only missing linear combination of "narrow"-band data. We included it because it displays the largest amplitude of variations for several stars.

Data relative to the programme stars are given in Table 1. Comparison stars are also listed, as is the number of useful observations. The "Rns" number refers to the catalogue of Ap and Am stars by Renson et al. (1991). One additional star, HD $190422=$ HR 7674, had been observed as a comparison and found to be variable.

\section{Analysis of the variations}

Differential data were calculated only when the time interval between the measurements between the comparison(s) and the programme star was short enough, usually well under 15 minutes. It was found that the differential data consistently provided slightly cleaner light curves than the absolute data.

Ap stars are normally characterized by very regular periodic variations which can be fitted by simple Fourier series (see Manfroid \& Renson 1994)

$m(t)=A_{s}+\sum_{i=1}^{I} B_{i} \cos \left(\frac{2 \pi i\left(t-t_{0}\right)}{P}+\phi_{i}\right)$ 
Table 1. Stars analyzed. Rns stands for the Catalogue Général des Etoiles Ap et $A m$ by Renson (Renson et al. 1991). The $V$ magnitude is an average derived from our Geneva measurements. The spectral type is from Renson's catalogue. The HD number of the comparison(s) is given. $N$ is the number of useful measurements

\begin{tabular}{|c|c|c|c|c|}
\hline HD & Rns & sp. type & comparison(s) & $N_{\text {obs }}$ \\
\hline $8717^{1}$ & 2100 & $8.358 \mathrm{~A} 4 \mathrm{Cr}$ & 9906 & 24 \\
\hline 9050 & 2194 & 8.952 A0 EuSr & 8589 & 25 \\
\hline $9529^{1}$ & 2310 & 9.164 F6 Sr & 14287 & 25 \\
\hline 187039 & 51550 & $9.396 \mathrm{~A} 2 \mathrm{Si}$ & $162515 \& 187923$ & 18 \\
\hline 187752 & 51800 & 8.503 B9 Si & $162515 \& 187923$ & 18 \\
\hline 188309 & 52010 & $9.968 \mathrm{~A} 2 \mathrm{Si}$ & $162515 \& 187923$ & 18 \\
\hline 191439 & 53340 & 8.887 B9 CrEuSr & & 29 \\
\hline $191857^{1}$ & 53500 & $8.805 \mathrm{~A} 1 \mathrm{Si}$ & $162515 \& 187923$ & 16 \\
\hline 355163 & 53540 & $9.628 \mathrm{~B} 9 \mathrm{Si}$ & $162515 \& 187923$ & 15 \\
\hline 192674 & 53730 & 7.552 B9 CrEuSr & & 29 \\
\hline 192687 & 53770 & $9.225 \mathrm{~A} 1 \mathrm{Si}$ & $162515 \& 187923$ & 18 \\
\hline 194750 & 54310 & 8.876 A0 $\mathrm{SrSiCr}$ & 199288 & 26 \\
\hline 195112 & 54390 & 8.847 B9 EuCrSr & 199288 & 28 \\
\hline $200199^{1}$ & 55760 & $6.998 \mathrm{~A} 1 \mathrm{Cr}$ & 201901 & 22 \\
\hline 200623 & 55940 & 9.066 A2 $\mathrm{SrEuCr}$ & 201901 & 21 \\
\hline $204367^{1}$ & 56910 & $7.805 \mathrm{~A} 0 \mathrm{SrEuCr}$ & 201901 & 21 \\
\hline 207259 & 57690 & 8.862 A0 EuCrSr & 203608 & 24 \\
\hline 216965 & 59900 & $8.317 \mathrm{~F} 0 \mathrm{p}$ & 218045 & 19 \\
\hline 222561 & 61020 & $8.519 \mathrm{~A} 4 \mathrm{p}$ & 222847 & 24 \\
\hline 222638 & 61030 & $8.662 \mathrm{~A} 0 \mathrm{SrEuCr}$ & 212330 & 26 \\
\hline 222925 & 61140 & $9.005 \mathrm{~F} 8 \mathrm{SrEu}$ & 212330 & 26 \\
\hline
\end{tabular}

1 Ap nature given as uncertain in Renson's catalogue.

Table 2. Periodicities deduced from the measurements. Alternative values of the period are given in cases where it is not possible to choose between aliases. The range is the total amplitude of the analytical curve which shows the largest variations. It is immediately followed by the number $i$ of that curve. $S / N$ is the signal-to-noise ratio for the light curve where it is the largest, and is followed by the number $j$ of that curve. It is the ratio of the total range divided by the standard deviation of the observation about the analytical curve

\begin{tabular}{lllllll}
\hline HD & \multicolumn{2}{l}{ Period (error) aliases } & range $i$ & $\mathrm{~S} / \mathrm{N}$ & $j$ \\
\hline 8717 & $2.15(0.01)$ & 0.685 & 0.0427 & 13 & 8.07 & 1 \\
188309 & $0.905(0.01)$ & $10.0 / 1.115$ & 0.0714 & 8 & 8.08 & 8 \\
191439 & $3.24(0.03)$ & & 0.0480 & 11 & 8.38 & 9 \\
192674 & $4.45(0.05)$ & $0.82 / 1.28 / 8.9$ & 0.0258 & 8 & 4.45 & 10 \\
192687 & $1.19(0.01)$ & 6.25 & 0.09218 & 10.21 & 8 \\
194750 & $2.26(0.01)$ & $0.688 / 4.48$ & 0.04338 & 5.63 & 10 \\
195112 & $1.82(0.01)$ & & 0.05618 & 8.25 & 8 \\
200623 & $2.18(0.01)$ & $0.684 / 1.85$ & 0.0526 & 17 & 11.68 & 17 \\
207259 & $2.16(0.01)$ & $0.681 / 4.31$ & 0.0527 & 17 & 13.77 & 17 \\
\hline
\end{tabular}

Table 3. Code to the Geneva magnitudes or indices

\begin{tabular}{ll}
\hline 1 & $V$ \\
2 & $U-B$ \\
3 & $V-B$ \\
4 & $B 1-B$ \\
5 & $B 2-B$ \\
6 & $V 1-B$ \\
7 & $G-B$ \\
8 & $U$ \\
9 & $B$ \\
10 & $B 1$ \\
11 & $B 2$ \\
12 & $V 1$ \\
13 & $G$ \\
14 & $B 1-B 2$ \\
15 & $B 2-V 1$ \\
16 & $V 1-G$ \\
17 & $B 1-V 1$ \\
\hline
\end{tabular}

where $m$ is the magnitude, $P$ the fundamental period, $I$ the total number of harmonics, $t$ the time and $t_{0}$ the origin of time.

A nice fit is generally obtained with two components, though a few stars are known to need 3 components e.g., 52 Oph, 27G. Car, 25 Sex, $\alpha$ Scl (Manfroid \& Renson 1994) and TW Col (Renson \& Manfroid 1992a) - or even 4 components as 46 Eri (Manfroid \& Renson 1989) and VV Scl (Renson \& Manfroid 1992b).

In all cases where high-order series have been derived, the third- or fourth-order components are small and a large number of accurate measurements were necessary to bring them out. On the other hand, the fundamental and the first overtone are larger and often of comparable amplitudes. In those rare instances where variations can be adequately described with a single cosine, it is often the first overtone which dominates the fundamental, yielding a symmetrical double wave (see, e.g., HD 83366, Mathys et al. 1985). These considerations show that a twocomponent series is well suited to analyze our data sets. The number of observations per free parameter in Eq. (1) is between 3 and 5 , which is adequate.

The parameters of the light curve were obtained by a least-squares method. The value of the period itself was estimated through two usual methods: (1) by looking for the minimum of the chi-square merit function over a set of trial periods (see, e.g., Manfroid \& Renson 1996; Manfroid \& Mathys 1997), and (2) by using the period-searching algorithm proposed by Renson $(1978,1980)$. The final values were checked by visual inspection of the phase diagrams.

As expected from measurements taken every night at about the same hour the periodograms are strongly affected by aliasing phenomena. We found convenient to compare those periodograms with those of simulated data 
Table 4. Parameters of the least-squares fits for HD 8717 with a period of 2 d 165 (see Eq. (1)). The error on each parameter is indicated in parentheses. $\sigma$ is the scatter around the least-squares fit. $r$ is the total range of the analytical light curve. $S / N$ is the signal-to-noise ratio defined as the total range divided into the scatter. Similar tables for the other stars are available from the CDS archive

\begin{tabular}{llllllll}
\hline & & HD 8717 & \multicolumn{2}{l}{$P=2.15$} & \multicolumn{2}{l}{$t_{0}=2450000.000$} \\
& $B_{1}$ & $B_{2}$ & $\phi_{1}$ & $\phi_{2}$ & $\sigma$ & $r$ & $\mathrm{~S} / \mathrm{N}$ \\
& & & & & & \\
1 & $0.0130(0.0017)$ & $0.0051(0.0018)$ & $4.00(0.13)$ & $5.92(0.32)$ & 0.0038 & 0.0309 & 8.07 \\
2 & $0.0049(0.0013)$ & $0.0051(0.0014)$ & $2.67(0.25)$ & $0.40(0.25)$ & 0.0058 & 0.0175 & 3.03 \\
3 & $0.0078(0.0013)$ & $0.0036(0.0014)$ & $4.02(0.16)$ & $5.87(0.35)$ & 0.0040 & 0.0193 & 4.84 \\
4 & $0.0027(0.0013)$ & $0.0008(0.0014)$ & $1.44(0.37)$ & $0.45(1.52)$ & 0.0040 & 0.0059 & 1.50 \\
5 & $0.0028(0.0017)$ & $0.0028(0.0018)$ & $3.52(0.57)$ & $0.02(0.46)$ & 0.0044 & 0.0094 & 2.14 \\
6 & $0.0077(0.0013)$ & $0.0047(0.0014)$ & $3.83(0.17)$ & $5.11(0.27)$ & 0.0041 & 0.0205 & 5.01 \\
7 & $0.0102(0.0017)$ & $0.0080(0.0018)$ & $4.12(0.16)$ & $0.05(0.16)$ & 0.0056 & 0.0314 & 5.66 \\
8 & $0.0080(0.0026)$ & $0.0063(0.0027)$ & $3.34(0.27)$ & $0.26(0.36)$ & 0.0067 & 0.0225 & 3.34 \\
9 & $0.0052(0.0021)$ & $0.0015(0.0023)$ & $3.97(0.41)$ & $6.04(1.25)$ & 0.0031 & 0.0117 & 3.76 \\
10 & $0.0033(0.0017)$ & $0.0022(0.0018)$ & $3.49(0.47)$ & $0.00(0.56)$ & 0.0041 & 0.0093 & 2.29 \\
11 & $0.0078(0.0021)$ & $0.0042(0.0023)$ & $3.81(0.27)$ & $6.21(0.39)$ & 0.0054 & 0.0208 & 3.81 \\
12 & $0.0129(0.0013)$ & $0.0057(0.0014)$ & $3.89(0.10)$ & $5.32(0.23)$ & 0.0048 & 0.0309 & 6.47 \\
13 & $0.0153(0.0013)$ & $0.0094(0.0014)$ & $4.07(0.08)$ & $0.00(0.10)$ & 0.0053 & 0.0427 & 8.05 \\
14 & $0.0047(0.0017)$ & $0.0020(0.0018)$ & $0.90(0.36)$ & $2.99(0.70)$ & 0.0053 & 0.0116 & 2.20 \\
15 & $0.0052(0.0013)$ & $0.0045(0.0014)$ & $0.85(0.25)$ & $1.36(0.26)$ & 0.0054 & 0.0157 & 2.91 \\
16 & $0.0035(0.0013)$ & $0.0077(0.0014)$ & $1.65(0.27)$ & $3.80(0.17)$ & 0.0061 & 0.0199 & 3.28 \\
17 & $0.0099(0.0017)$ & $0.0048(0.0018)$ & $0.88(0.17)$ & $1.79(0.32)$ & 0.0061 & 0.0223 & 3.67 \\
\hline
\end{tabular}

including a single periodic signal. Simulations were built for each of the suspected periods, and they were sampled exactly as the actual data. This provided a help for discarding spurious periods. There are nevertheless several stars for which we could not make unambiguous choice.

More than half the stars have shown clear variations within the time span of the observations. For nine stars, periodicities could be derived. The remaining stars have either too low an amplitude, or too long a period.

Table 2 lists the periods obtained (with plausible aliases for some stars) as well as some major characteristics of the variations: (1) the total amplitude in the most variable of the 17 lightcurves, as derived from the analytical model, and (2) the ratio of the total amplitude divided by the standard deviation of the residuals in the magnitude or color index where this ratio is the largest. The latter quantity shows which of the 17 lightcurves is the cleanest. (Our coding of the Geneva magnitudes or indices is given in Table 3 ).

Beside these 9 stars, HD 9529, 187039, 187752, 191857 and 222561, showed signs of variability, but no definite period could be found.

The detailed tables containing all the parameters of the lightcurves are stored in digital form at the CDS. HD 8717 is presented in Table 4 as an example.

A few selected lightcurves are presented in Fig. 1. All curves $(17 \times 9)$ are also to be found at the CDS.

\section{Discussion}

Although they are all standard of the Geneva system, we checked the absolute photometry of each comparison star in order to detect possible spurious phenomena. All were perfectly stable, with the exception of HD 190422, comparison star of HD 191439 and HD 192674, which was found to be slightly variable with a period of about 3 days. With a spectral type F8V, HD 190422 may be a small-amplitude cepheid. A more detailed analysis using additional data is being carried out.

The absolute data of HD 191439 and HD 192674 were analyzed instead of the differential ones. Variations could be detected in the former, with a period of three days, close to that of HD 190422. Because of this coincidence, a blind analysis of the differential data would not have raised any suspicion about the comparison. The period of HD 192674 is less clear. We favor the value $P=4$. 45 .

Another coincidence jumps to the eyes. It was immediately apparent during the analysis that an uncomfortably large number of stars had similar periodograms, with peaks at the same positions (see Fig. 1). It is well known that the distribution of the periods of Ap stars has a maximum between 2 and 2.5 days, but it was hard to believe that three stars in such a small sample could have almost exactly the same period of 2 d 16 ! We initially suspected some flaw in the measurement or reduction procedures. However, other stars measured in almost exactly the same conditions (comparisons, or nearby Ap stars) did not show the same behaviour. Moreover, the phase diagrams 

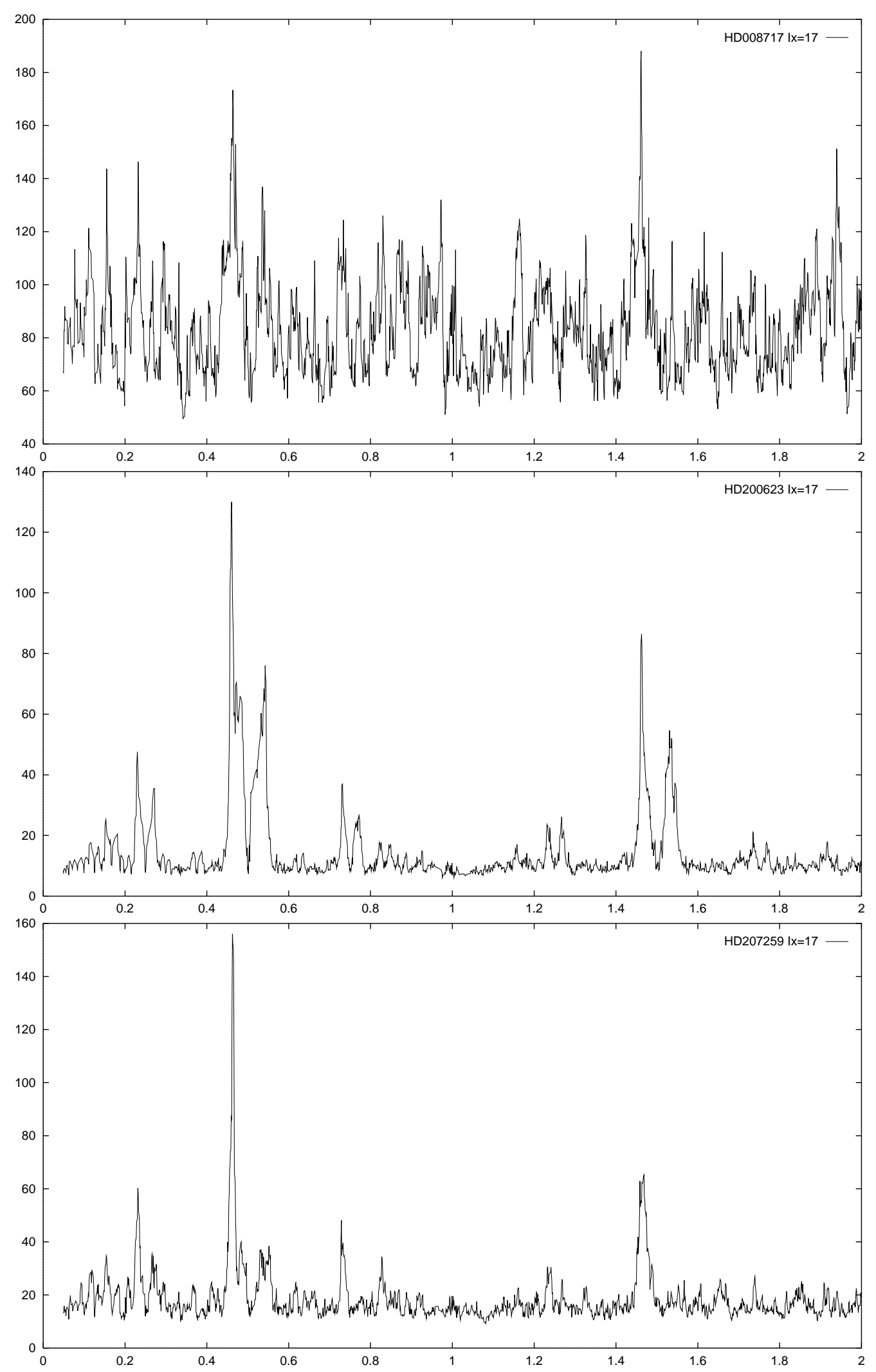

Fig. 1. Periodogrammes for the differential data of HD 8717, 200623 and 207259 in $B 1-V 1$. The parameter plotted is Renson's $1 / \theta_{1}$. The horizontal axis is the frequency in $\mathrm{d}^{-1}$. These stars have the peculiarity of showing almost exactly the same period $P=2$. 16 . The noise level and the relative importance of the various aliases differ from star to star. The complete set of periodogrammes is available at the CDS 

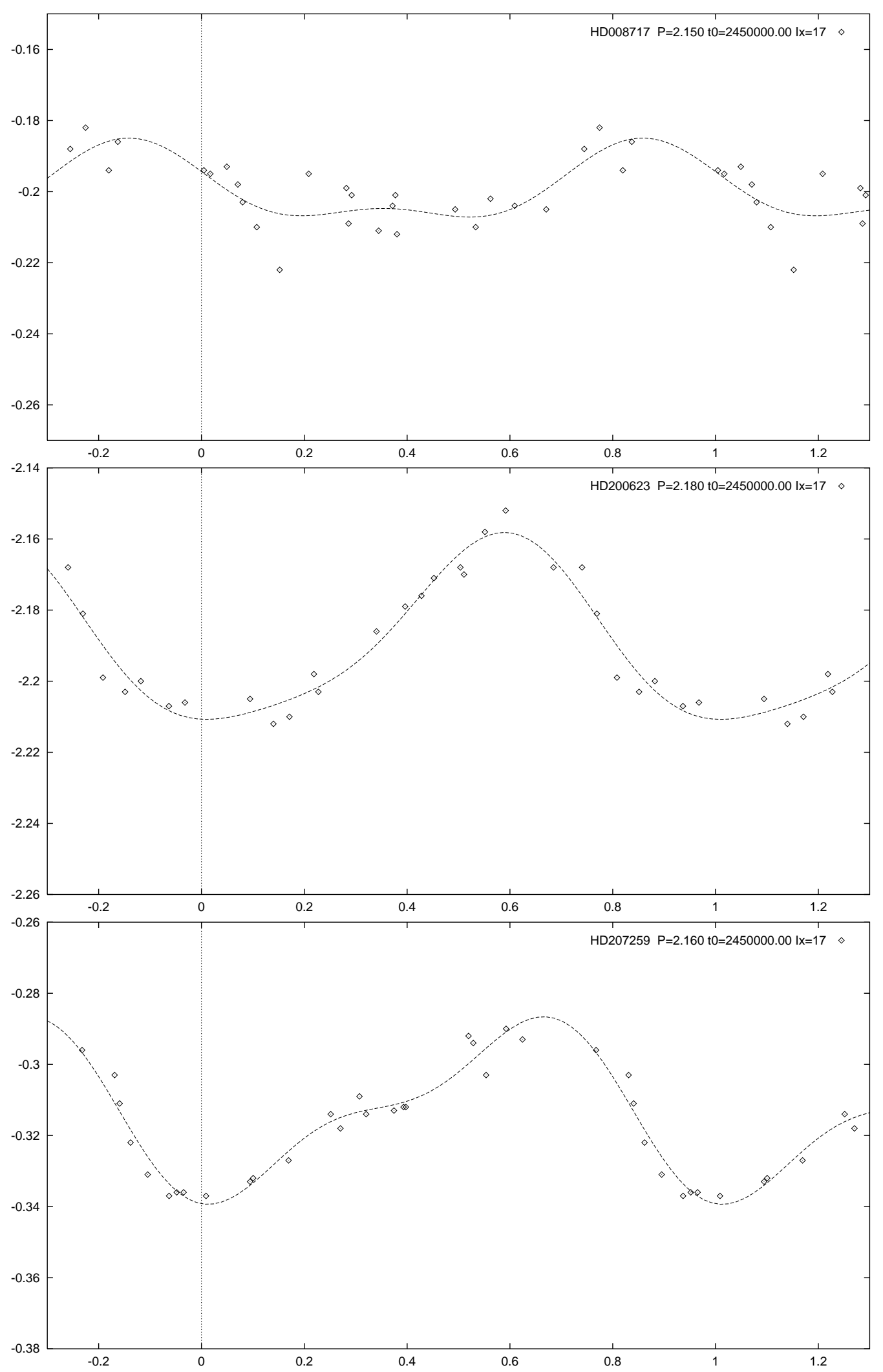

Fig. 2. Phase diagrams for the differential data of HD 8717, 200623 and 207259 in $B 1-V 1$. Time origin is JD 24500000 . The curves are second-order Fourier series. These stars have the peculiarity of showing almost exactly the same period $P=2$. 16 (see Fig. 1). Tick marks on the vertical axis are separated by 0.01 mag. The complete set of lightcurves is available at the CDS 
constructed with these periods were quite different for the various stars (see Fig. 2). We were then forced to admit the possibility of those coincidences. Aside from these three stars, a fourth one has a slightly longer one, and a fifth one has almost the double period. Looking at older data, we found that coincidences abound in Ap stars studies. In an earlier study of 56 stars, Mathys \& Manfroid (1985) found three stars with a period within 0.01 of 2.20 and two stars with the longer period 2 d 23 .

Among the Ap stars classified as uncertain by Renson (1991), HD 8717 shows Ap-like variations, which would confirm its status in this category.

The most uncertain cases in Table 2 are HD 192674 and HD 192687. The proposed periods are only tentative. There are no indication of variations in the suspected Ap stars 200199 and 204367.

HD 191439 and HD 200623 had already been observed by Vogt \& Faundez (1979). From four uvby measurements these authors could not recognize the variability of both stars.

Inspection of the lightcurves, and Table 2, show that, despite the weaker signals, magnitudes and color indices involving narrow-band filters often provide cleaner light curves, a conclusion already reached from the comparison of Strömgren's and Johnson's photometries. In this respect, the neglected $B 1-V 1$ index proves to be interesting. We also note that the largest amplitudes often occur in the $U$ band, which is a well-known characteristics of Ap stars.
Acknowledgements. We are grateful to the Geneva Observatory and especially to Pr. G. Burki for making possible these observations at the Swiss telescope on La Silla. Our thanks also go to Mr. C. Richard and Mr. B. Pernier who carried out the data reductions.

\section{References}

Burnet M., Rufener F., 1979, A\&A 74, 54

Maitzen H.M., 1976, A\&A 51, 223

Manfroid J., Mathys G., 1985, A\&AS 59, 429

Manfroid J., Mathys G., 1997, A\&AS (in press)

Manfroid J., Renson P., 1989, A\&A 223, 187

Manfroid J., Renson P., 1994, A\&A 281, 73

Mathys G., Manfroid J., 1985, A\&AS 60, 17

North P., 1984, A\&AS 55, 259

North P., Adelman S.J., 1995, A\&AS 111, 41

Renson P., 1978, A\&A 63, 125

Renson P., 1980, A\&A 92, 30

Renson P., Gerbaldi M., Catalano F.A., 1991, A\&AS 89, 429

Renson P., Manfroid J., 1992a, A\&A 256, 104

Renson P., Manfroid J., 1992b, A\&A 263, 161

Rufener F., 1988, Catalogue of Stars measured in the Geneva Observatory Photometric system, fourth edition, Obs. de Genève

Vogt N., Faundez M., 1979, A\&AS 36, 477 